PROCEEDINGS OF THE

AMERICAN MATHEMATICAL SOCIETY

Volume 129, Number 12, Pages 3671-3681

S 0002-9939(01)06316-X

Article electronically published on July 10, 2001

\title{
EXCHANGE OF CONSERVED QUANTITIES IN NONHYPERBOLIC SYSTEMS-AN EXAMPLE
}

\author{
MICHAEL SEVER
}

(Communicated by Suncica Canic)

\begin{abstract}
The velocity function associated with a smooth solution of "zero pressure gas dynamics" satisfies Burgers equation. Indeed an elementary generalization holds for scalar conservation laws. Weak solutions, however, are compatible in this respect only under special conditions on the initial density function. Our result depends on the uniform convexity of the flux function associated with the scalar equation, and on the entropy condition applied to both systems.
\end{abstract}

\section{INTRODUCTION}

Smooth solutions of systems of conservation laws

$$
w_{t}+h(w)_{x}=0
$$

where $w(x, t) \in \mathbb{R}^{n}$ and $h$ is a smooth map of $\mathbb{R}^{n}$ into $\mathbb{R}^{n}$, satisfy extensions of the form

$$
U(w)_{t}+F(w)_{x}=0
$$

provided that the scalar functions $U, F$ satisfy $\left|U_{w}(w)\right|$ bounded and $U_{w}(w) f_{w}(w)=$ $F_{w}(w)$ for all $w$.

For systems (1.1) which are hyperbolic, for example with $U$ convex in $w$ [4, and for some systems which change type [10, the existence of such extensions has two well-known applications. Admissible weak solutions are characterized by an inequality replacing (1.2) 7]. Alternatively piecewise smooth weak solutions of (1.1) satisfy (1.2) up to third order in the "shock strength", admitting the possibility of replacing one of the constituent equations in (1.1) by the extensions (1.2) [5, 12]. One thus obtains a closely related approximation to (1.1) which may be expedient for computational purposes, for example the adiabatic approximation of the Euler equations of gas dynamics.

The proof that weak solutions of (1.1) containing only shocks of modest strength approximately satisfy (1.2) does not depend on hyperbolicity of (1.1) or on any special properties of $U$, but on the assumed form of a weak solution, a piecewise smooth, bounded measurable function in particular, and on the existence of a smooth Hugoniot locus connecting the limiting values of $w$ at points of discontinuity. In this spirit

Received by the editors May 1, 2000.

2000 Mathematics Subject Classification. Primary 35L65, 35L67.

This research was partially supported by the Texas Advanced Research Program under grant 00365-2102-ARP. 
we address the question of whether weak solutions of (1.1) satisfy or approximately satisfy (1.2) weakly when the system (1.1) admits real characteristic speeds but fails to be hyperbolic because of a deficiency of eigenvectors. In this case the limiting values of $w$ at a shock do not in general satisfy a Rankine-Hugoniot relation of the usual form 9 , 13.

Relations between scalar conservation laws and pairs with equal characteristic speeds are well established [2, 6].

For example, smooth solutions of a simple generalization of one-dimensional "zero pressure gas dynamics"

$$
\begin{aligned}
& \rho_{t}+\left(\rho f^{\prime}(u)\right)_{x}=0, \\
& (\rho u)_{t}+\left(\rho u f^{\prime}(u)\right)_{x}=0,
\end{aligned}
$$

with $\rho(x, t), u(x, t)$ scalar-valued, satisfy a scalar conservation law independent of $\rho$,

$$
u_{t}+f(u)_{x}=0
$$

Thus given initial data for $\rho, u$, one is motivated to find $u$ from (1.5) and then $\rho$ from (1.3). However, (1.4) will be (weakly) satisfied or approximately satisfied only under special conditions on the initial data; results to this effect follow. The underlying problem is that weak solutions of (1.3), (1.4) are not bounded, but contain "delta-shocks", with $\rho$ a distribution and $u$ discontinuous [1, 3, 9].

\section{MAIN THEOREM}

We shall assume the flux function $f$ in (1.3)-(1.5) to be smooth and uniformly convex in $u$ throughout, taking $f^{\prime \prime}>0$ for definiteness. A piecewise smooth admissible weak solution of $(1.5)$ in $\mathbb{R} \times[0, \tau]$ will then be characterized by distinct shock curves $\Gamma_{k}=\left\{\left(x_{k}(t), t\right), t \in I_{k} \subset(0, \tau]\right\}, k=1, \ldots$, originating at points where shocks form spontaneously or at points $Q_{j}, j=1, \ldots$, where two or more shocks collide, and ending at points where shocks collide or at $t=\tau$. For simplicity we shall assume $u(\cdot, 0)$ continuous, but this can readily be relaxed.

The shock speed $s_{k}(t)=d x_{k}(t) / d t$ (forward time derivative) is obtained from the Rankine-Hugoniot condition

$$
s_{k}(t)=\frac{f\left(u\left(x_{k}(t)-0, t\right)\right)-f\left(u\left(x_{k}(t)+0, t\right)\right)}{u\left(x_{k}(t)-0, t\right)-u\left(x_{k}(t)+0, t\right)}
$$

and satisfies the entropy condition

$$
f^{\prime}\left(u\left(x_{k}(t)-0, t\right)\right)>s_{k}(t)>f^{\prime}\left(u\left(x_{k}(t)+0, t\right)\right),
$$

equivalently

$$
u\left(x_{k}(t)-0, t\right)>\zeta\left(s_{k}(t)\right)>u\left(x_{k}(t)+0, t\right),
$$

where in (2.3) and below we abbreviate $f^{\prime-1}$ by $\zeta$.

The definition of a weak solution of (1.3), (1.4) is complicated by the anticipated low regularity, and is discussed in [11. The reader may wish to focus on the case $f=\frac{1}{2} u^{2}$, recovering zero pressure gas dynamics and Burgers equation, respectively, from (1.3), (1.4) and (1.5), and making both $f^{\prime}$ and $\zeta$ the identity function.

A weak solution of (1.3), (1.4) is determined by a Lagrange function $X: \mathbb{R} \times$ $[0, \tau] \rightarrow \mathbb{R}$ and a positive measure $d \mu$ on $\mathbb{R}$. For each $y \in \mathbb{R}, t \in(0, \tau]$ we identify 
$X(y, t)$ as the position of a particle initially at the point $X(y, 0)$. For $u(\cdot, 0)$ continuous, we may take $X(y, 0)=y$, and identify the initial density $\rho(\cdot, 0)$ with the Radon-Nikodym derivative

$$
\frac{d \mu(x)}{d x}=\rho(x, 0), x \in \mathbb{R} .
$$

More generally, the moments of $\rho$ are obtained from

$$
\iint \theta(x, t) \rho(x, t) d x d t=\iint \theta(X(y, t), t) d \mu(y) d t
$$

for $\theta$ continuous and of compact support.

Denote by

$$
\begin{aligned}
K= & \left\{\phi \in L^{\infty}(\mathbb{R} \times[0, \tau] \rightarrow \mathbb{R}) \mid\right. \\
& \iint \phi^{2}(y, t) d \mu(y) d t<\infty \text { and } \\
& \phi(y, t)=\theta(X(y, t), t), \quad \text { for some } \theta \in L^{\infty}(\mathbb{R} \times[0, \tau] \rightarrow \mathbb{R}) \\
& \text { a.e. in } \mathbb{R} \times[0, \tau]\} .
\end{aligned}
$$

Definition. $X, d \mu$ determine a weak solution of (1.3), (1.4) in $\mathbb{R} \times[0, \tau]$ if $X_{t} \in$ $K$ ( $X_{t}$ is the forward time derivative at constant $y$ ) and if, for all $\theta$ continuous and of compact support in $\mathbb{R} \times[0, \tau]$,

$$
\iint \zeta\left(X_{t}(y, t)\right)_{t} \theta(X(y, t), t) d \mu(y) d t=0 .
$$

This definition is based on a definition obtained in [11. An alternative, indeed simpler, definition of a weak solution of (1.3), (1.4) was given in [3], but is not used here.

Definition. An entropy weak solution of $(1.3),(1.4)$ is a weak solution for which $X$ satisfies an additional condition $X\left(y_{1}, t\right)=X\left(y_{2}, t\right)$ for all $y_{1} \neq y_{2}, t>t_{0}$ such that $X\left(y_{1}, t_{0}\right)=X\left(y_{2}, t_{0}\right)$.

For given initial data, the uniqueness of an entropy weak solution is discussed in [3, 8].

Definition. A piecewise smooth weak solution $u$ of (1.5) is compatible with a weak solution $X, d \mu$ of (1.3), (1.4) if

$$
u(x, t)=\zeta\left(X_{t}(y, t)\right), \quad \text { equivalently } f^{\prime}(u(x, t))=X_{t}(y, t),
$$

for all $x=X(y, t)$ such that $u$ is continuous at $(x, t)$.

If $u$ is a piecewise smooth admissible weak solution of (1.5) and $X, d \mu$ determine an entropy weak solution of (1.3), (1.4), then in both cases the characteristic(s) from any point $x, t$ continue(s) backwards in time to the initial line $t=0$ without intersecting a shock curve. In addition $u$ and $X_{t}$ are constant on the respective characteristics. So an admissible weak solution of (1.5) is compatible with an entropy weak solution of (1.3), (1.4) if the initial velocities are equal,

$$
f^{\prime}(u(x, 0))=X_{t}(x, 0), x \in \mathbb{R},
$$

and if the respective shock curves coincide.

Such compatible solutions satisfy (1.3), (1.4), (1.5) (weakly) simultaneously, with the above definition of a weak solution of (1.3), (1.4). 
Theorem 2.1. Given an initial velocity $u(\cdot, 0)$ of class $C^{1}$ and of compact support and any $\tau>0$, there exists a positive, bounded initial density $\rho(\cdot, 0)$ such that the admissible weak solution of (1.5) in $\mathbb{R} \times[0, \tau]$ is compatible with the corresponding entropy weak solution of (1.3), (1.4).

Proof. Let $u$ denote the unique admissible weak solution of (1.5). We determine $X$ from

$$
\begin{aligned}
& X(y, 0)=y \\
& X_{t}(y, t)=f^{\prime}(u(X(y, t), t)), \quad u \text { continuous at }(X(y, t), t) \\
& X_{t}(y, t)=s_{k}(t),(X(y, t), t) \in \Gamma_{k}, k=1, \ldots
\end{aligned}
$$

This choice makes the shock curves coincide, assures $X_{t} \in K$ and that (2.8) holds. It remains to determine $\rho(\cdot, 0)$ (and $d \mu$ from $(2.4)$ ) such that $X, d \mu$ determine a weak solution of $(1.3),(1.4)$, i.e. such that $(2.7)$ is satisfied.

For $y, t$ such that $u$ is continuous at $(X(y, t), t)$,

$$
\begin{aligned}
\zeta\left(X_{t}(y, t)\right)_{t} & =\frac{\partial}{\partial t} u(X(y, t), t) \\
& =u_{t}(x, t)+X_{t}(y, t) u_{x}(x, t) \\
& =u_{t}(x, t)+f^{\prime}(u(x, t)) u_{x}(x, t) \\
& =0
\end{aligned}
$$

using (2.8) and (1.5), with $x=X(y, t)$ throughout. So the integral in (2.7) contains only contributions from the shock curves $\Gamma_{k}$ and the collision points $Q_{j}$ which depend in particular on the mass which accumulates on each of the "delta-shocks".

In (2.7), consider the case of a test function $\theta$ with support intersecting a single shock curve $\Gamma_{k}$ but none of the collision points $Q_{j}$. At each point $\left(x_{k}(t), t\right) \in \Gamma_{k}$, the limiting values of $\rho, u$ are

$$
\rho_{k}^{ \pm}(t)=\rho\left(x_{k}(t) \pm 0, t\right), u_{k}^{ \pm}(t)=u\left(x_{k}(t) \pm 0, t\right)
$$

From (1.5) $u$ is constant on the characteristics, which continue back to the initial line $t=0$ at points $z_{k}^{ \pm}(t)$ without intersecting any shock curves, so

$$
u_{k}^{ \pm}(t)=u\left(z_{k}^{ \pm}(t), 0\right)
$$

with $z_{k}^{ \pm}(t)$ given by

$$
z_{k}^{ \pm}(t)=x_{k}(t)-t f^{\prime}\left(u_{k}^{ \pm}(t)\right)
$$

The speed of the shock $s_{k}(t)$ is obtained from (2.1), and the accumulated mass $m_{k}(t)$ is given by

$$
m_{k}(t)=\int_{z_{k}^{-}(t)}^{z_{k}^{+}(t)} \rho(x, 0) d x
$$


In view of (2.13) for such $\theta$ the integral in (2.7) is

$$
\begin{aligned}
& \lim _{\varepsilon \downarrow 0} \int_{I_{k}} \int_{\mu\left(z_{k}^{-}(t)-\varepsilon\right)}^{\mu\left(z_{k}^{+}(t)+\varepsilon\right)} \theta(X(y, t), t) \zeta\left(X_{t}(y, t)\right)_{t} d \mu(y) d t \\
& =\lim _{\varepsilon \downarrow 0} \int_{I_{k}} \theta\left(x_{k}(t), t \int_{\mu\left(z_{k}^{-}(t)-\varepsilon\right)}^{\mu\left(z_{k}^{+}(t)+\varepsilon\right.} \zeta\left(X_{t}(y, t)\right)_{t} d \mu(y) d t+O(\varepsilon)\right. \\
& =\lim _{\varepsilon \downarrow 0} \int_{I_{k}} \theta\left(x_{k}(t), t\right) \int_{z_{k}^{+}(t)+\varepsilon}^{z^{-}(t)-\varepsilon} \zeta\left(X_{t}(y, t)\right)_{t} \rho(y, 0) d y d t+O(\varepsilon) \\
& =-\lim _{\varepsilon \downarrow 0} \int_{I_{k}} \frac{d}{d t} \theta\left(x_{k}(t), t\right) \int_{z_{k}^{-}(t)-\varepsilon} \zeta\left(X_{t}(y, t)\right) \rho(y, 0) d y d t \\
& -\lim _{\varepsilon \downarrow 0} \int_{I_{k}} \theta\left(x_{k}(t), t\right)\left[\zeta\left(X_{t}\left(z_{k}^{+}(t)+\varepsilon, t\right)\right) \rho\left(z_{k}^{+}(t)+\varepsilon, 0\right) z_{k, t}^{+}(t)\right. \\
& \left.-\zeta\left(X_{t}\left(z_{k}^{-}(t)-\varepsilon, t\right)\right) \rho\left(z_{k}^{-}(t)-\varepsilon, 0\right) z_{k, t}^{-}(t)\right] d t+O(\varepsilon),
\end{aligned}
$$

using the continuity of $\theta$, the definition of $\mu$ in (2.4), and a partial integration.

From (2.12)

$$
X_{t}(y, t)=s_{k}(t), z_{k}^{-}(t)<y<z_{k}^{+}(t)
$$

whereas

$$
\lim _{\varepsilon \downarrow 0} X_{t}\left(z_{k}^{ \pm}(t) \pm \varepsilon\right)=f^{\prime}\left(u_{k}^{ \pm}(t)\right)
$$

Taking the limit as $\varepsilon \downarrow 0$ in (2.18), using (2.17), (2.19), (2.20), we obtain the integral in (2.7) equal to

$$
\begin{aligned}
& -\int_{I_{k}} \frac{d}{d t} \theta\left(x_{k}(t), t\right) \zeta\left(s_{k}(t)\right) m_{k}(t) d t \\
& \quad-\int_{I_{k}} \theta\left(x_{k}(t), t\right)\left[u_{k}^{+}(t) \rho\left(z_{k}^{+}(t), 0\right) z_{k, t}^{+}(t)-u_{k}^{-}(t) \rho\left(z_{k}^{-}(t), 0\right) z_{k, t}^{-}(t)\right] d t \\
& \quad=\int_{I_{k}} \theta\left(x_{k}(t), t\right) M_{k}(t) d t
\end{aligned}
$$

where

$$
M_{k}(t)=\frac{d}{d t}\left(\zeta\left(s_{k}(t)\right) m_{k}(t)\right)-u_{k}^{+}(t) \rho\left(z_{k}^{+}(t), 0\right) z_{k, t}^{+}(t)+u_{k}^{-}(t) \rho\left(z_{k}^{-}(t), 0\right) z_{k, t}^{-}(t) .
$$

For (2.7) to hold for such $\theta$, it suffices that $M_{k}(t)$ given by $(2.22)$ vanish for almost all $t \in I_{k}$.

Differentiating (2.17) with respect to time,

$$
m_{k, t}(t)=\rho\left(z_{k}^{+}(t), 0\right) z_{k, t}^{+}(t)-\rho\left(z_{k}^{-}(t), 0\right) z_{k, t}^{-}(t) .
$$


Differentiating (2.1) with respect to $t$ we find

$$
\begin{aligned}
\frac{d}{d t} \zeta\left(s_{k}\right)= & \frac{1}{f^{\prime \prime}\left(\zeta\left(s_{k}\right)\right)} \frac{d s_{k}}{d t} \\
= & \frac{1}{f^{\prime \prime}\left(\zeta\left(s_{k}\right)\right)}\left[\frac{f^{\prime}\left(u_{k}^{-}\right)\left(u_{k}^{-}-u_{k}^{+}\right)-f\left(u_{k}^{-}\right)+f\left(u_{k}^{+}\right)}{\left(u_{k}^{-}-u_{k}^{+}\right)^{2}} \frac{d}{d t} u_{k}^{-}\right. \\
& \left.\quad-\frac{f^{\prime}\left(u_{k}^{+}\right)\left(u_{k}^{-}-u_{k}^{+}\right)+f\left(u_{k}^{+}\right)-f\left(u_{k}^{-}\right)}{\left(u_{k}^{-}-u_{k}^{+}\right)^{2}} \frac{d}{d t} u_{k}^{+}\right] .
\end{aligned}
$$

From (1.5), the limiting values $u_{k}^{ \pm}$satisfy

$$
\begin{aligned}
u_{k}^{ \pm}(t) & =u_{t}\left(x_{k}(t) \pm 0, t\right)+s_{k}(t) u_{x}\left(x_{k}(t) \pm 0, t\right) \\
& =\left(s_{k}(t)-f^{\prime}\left(u_{k}^{ \pm}(t)\right)\right) u_{x}\left(s_{k}(t) \pm 0, t\right) .
\end{aligned}
$$

Differentiating (2.16) with respect to time

$$
\begin{aligned}
z_{k, t}^{ \pm}(t) & =s_{k}(t)-f^{\prime}\left(u_{k}^{ \pm}(t)\right)-t f^{\prime \prime}\left(u_{k}^{ \pm}(t)\right) u_{k, t}^{ \pm}(t) \\
& =\left(s_{k}(t)-f^{\prime}\left(u_{k}^{ \pm}(t)\right)\right)\left(1-t f^{\prime \prime}\left(u_{k}^{ \pm}(t)\right) u_{x}\left(x_{k}(t) \pm 0, t\right)\right)
\end{aligned}
$$

using (2.25). Using (2.2), $z_{k, t}^{-}<0$ and $z_{k, t}^{+}>0$.

Finally using (1.5) and (2.15), the limiting values of $u_{x}$ may be related to values on the initial line

$$
u_{k, x}^{ \pm}(t)=\frac{u_{x}\left(z_{k}^{ \pm}(t), 0\right)}{1+t f^{\prime \prime}\left(u_{k}^{ \pm}(t)\right) u_{x}\left(z_{k}^{ \pm}(t), 0\right)} .
$$

Now using (2.23), (2.24), (2.25), (2.26), (2.27), in (2.22), we obtain an expression for $M_{k}(t)$, given by

$$
\begin{aligned}
& \left(\frac{f^{\prime}\left(u_{k}^{-}\right)-s_{k}}{1+t f^{\prime \prime}\left(u_{k}^{-}\right) u_{x}\left(z_{k}^{-}, 0\right)}\right) \\
& \cdot\left[-\frac{m_{k}}{f^{\prime \prime}\left(\zeta\left(s_{k}\right)\right)} \frac{\left(f^{\prime}\left(u_{k}^{-}\right)\left(u_{k}^{-}-u_{k}^{+}\right)-f\left(u_{k}^{-}\right)+f\left(u_{k}^{+}\right)\right)}{\left(u_{k}^{-}-u_{k}^{+}\right)^{2}} u_{x}\left(z_{k}^{-}, 0\right)\right. \\
& \left.\quad-\rho\left(z_{k}^{-}, 0\right)\left(u_{k}^{-}-\zeta\left(s_{k}\right)\right)\right]+\left(\frac{s_{k}-f^{\prime}\left(u_{k}^{+}\right)}{\left(1+t f^{\prime \prime}\left(u_{k}^{+}\right) u_{x}\left(z_{k}^{+}, 0\right)\right)}\right)\left[-\frac{m_{k}}{f^{\prime \prime}\left(\zeta\left(s_{k}\right)\right)}\right. \\
& \left.\quad \cdot \frac{\left(f^{\prime}\left(u_{k}^{+}\right)\left(u_{k}^{-}-u_{k}^{+}\right)+f\left(u_{k}^{+}\right)-f\left(u_{k}^{-}\right)\right)}{\left(u_{k}^{-}-u_{k}^{+}\right)^{2}} u_{x}\left(z_{k}^{+}, 0\right)+\rho\left(z_{k}^{+}, 0\right)\left(\zeta\left(s_{k}\right)-u_{k}^{+}\right)\right] .
\end{aligned}
$$

Using the strict convexity of $f$ and the entropy condition (2.2) or (2.3) in (2.28), (2.22) assumes a form

$$
\begin{aligned}
M_{k}(t) & =-A^{-} B^{-}+A^{+} B^{+}, \\
A^{-} & =\frac{f^{\prime}\left(u_{k}^{-}\right)-s_{k}}{1+t f^{\prime \prime}\left(u_{k}^{-}\right) u_{x}\left(z_{k}^{-}, 0\right)}, \\
B^{-} & =C^{-} m_{k} u_{x}\left(z_{k}^{-}, 0\right)+\rho\left(z_{k}^{-}, 0\right)\left(u_{k}^{-}-\zeta\left(s_{k}\right)\right), \\
A^{+} & =\frac{s_{k}-f^{\prime}\left(u_{k}^{+}\right)}{1+t f^{\prime \prime}\left(u_{k}^{+}\right) u_{x}\left(z_{k}^{+}, 0\right)}, \\
B^{+} & =\rho\left(z_{k}^{+}, 0\right)\left(\zeta\left(s_{k}\right)-u_{k}^{+}\right)+C^{+} m_{k} u_{x}\left(z_{k}^{+}, 0\right),
\end{aligned}
$$


where the $C^{ \pm}$, depending on $u_{k}^{ \pm}$and $s_{k}$, are positive, bounded above and below using the strict convexity of $f$ and the entropy condition (2.2) or (2.3).

From the entropy condition and the fact that the characteristics from $\left(z_{k}^{ \pm}, 0\right)$ reach the shock curve $\Gamma_{k}$, it follows that the factors $A^{ \pm}$in (2.29) are defined (except possibly on a set of measure zero in $I_{k}$ ) and positive.

The initial densities $\rho\left(z_{k}^{ \pm}(t), 0\right), t \in I_{k}$, can now be chosen, strictly positive and bounded, so that $M_{k}(t)$ given in (2.29) vanishes for almost all $t$, i.e. wherever $A^{ \pm}$ are defined. At each $t \in I_{k}$, the choice of $\rho\left(z_{k}^{ \pm}(t), 0\right)$ depends on the signs of the $u_{x}\left(z_{k}^{ \pm}(t), 0\right)$. We use $m_{k}(t) \geq 0$ throughout.

For $u_{x}\left(z_{k}^{ \pm}(t), 0\right)$ both negative or zero, $\rho\left(z_{k}^{ \pm}(t), 0\right)>0$ can be chosen so that $B^{+}$ and $B^{-}$vanish.

For $u_{x}\left(z_{k}^{+}(t), 0\right)>0$ but $u_{x}\left(z_{k}^{-}(t), 0\right) \leq 0, A^{+}$is bounded; choose $\rho\left(z_{k}^{+}(t), 0\right)>0$ otherwise arbitrary. From $(2.29), B^{+}>0$, so

$$
\rho\left(z_{k}^{-}, 0\right)=-\frac{C^{-} u_{x}\left(z_{k}^{-}, 0\right)}{u_{k}^{-}-\zeta\left(s_{k}\right)} m_{k}+\frac{A^{+} B^{+}}{A^{-}\left(u_{k}^{-}-\zeta\left(s_{k}\right)\right)}
$$

makes $M_{k}(t)$ vanish. The case of $u_{x}\left(z_{k}^{+}(t), 0\right) \leq 0, u_{x}\left(z_{k}^{-}(t), 0\right)>0$ is completely similar.

For $u_{x}\left(z_{k}^{ \pm}, 0\right)$ both positive, $(2.29)$ is rewritten as

$$
\begin{aligned}
M_{k}(t) & =\left(A^{+} C^{+} u_{x}\left(z_{k}^{+}, 0\right)-A^{-} C^{-} u_{x}\left(z_{k}^{-}, 0\right)\right) m_{k} \\
& -A^{-}\left(u_{k}^{-}-\zeta\left(s_{k}\right)\right) \rho\left(z_{k}^{-}, 0\right)+A^{+}\left(\zeta\left(s_{k}\right)-u_{k}^{+}\right) \rho\left(z_{k}^{+}, 0\right) .
\end{aligned}
$$

In (2.31), $A^{ \pm}$are now both bounded and the last two terms are of opposite sign. Therefore for the first right-hand factor in (2.31) positive (negative), choose $\rho\left(z_{k}^{+}, 0\right)>0\left(\rho\left(z_{k}^{-}, 0\right)>0\right)$ arbitrarily and find $\rho\left(z_{k}^{-}, 0\right)>0\left(\rho\left(z_{k}^{+}, 0\right)>0\right)$ so that the right side of $(2.31)$ vanishes.

From (2.29), the only way that the $\rho\left(z_{k}^{ \pm}, 0\right)$ obtained in this manner could fail to be bounded is that $m_{k} /\left(u_{k}^{-}-u_{k}^{+}\right)$is not bounded.

From (2.17), $m_{k}$ is bounded from above. As shocks in the solution of (1.5) never disappear in finite time, $m_{k} /\left(u_{k}^{-}-u_{k}^{+}\right)$is bounded except possibly in the neighborhood of a point $(\underline{x}, \underline{t})$ where $\Gamma_{k}$ originates.

From (2.15)

$$
\begin{aligned}
u_{k}^{-}(t)-u_{k}^{+}(t) & =u\left(z_{k}^{-}(t), 0\right)-u\left(z_{k}^{+}(t), 0\right) \\
& =-\int_{z_{k}^{-}(t)}^{z_{k}^{+}(t)} u_{x}(x, 0) d x
\end{aligned}
$$

and as the shock form after time $\underline{t}<\tau$, for $x$ in a neighborhood of $z_{k}^{ \pm}(\underline{t})$ we have

$$
-u_{x}(x, 0)>\frac{1}{\tau \sup f^{\prime \prime}(u)}
$$

for $u$ in a neighborhood of $u\left(z_{k}^{ \pm}(\underline{t}), 0\right)$.

Now from (2.17), (2.32), (2.33), for $t-\underline{t}$ small,

$$
\frac{m_{k}(t)}{u_{k}^{-}(t)-u_{k}^{+}(t)} \leq \tau(\sup \rho(x, 0))\left(\sup f^{\prime \prime}(u(x, 0))\right.
$$

for $x$ in a neighborhood of $z_{k}^{ \pm}(\underline{t})$. 
Finally, we consider (2.7) in the case where the support of $\theta$ includes a shock collision point $Q_{j}=(\bar{x}, \bar{t})$ with $k_{1}, \ldots, k_{n}$ denoting the $n$ colliding shocks and $j$ the single resulting shock, using (1.5).

Adopting the convention $x_{k_{i}}<x_{k_{i+1}}, i=1, \ldots, n-1$, and using (2.16) we have

$$
\begin{aligned}
& \bar{x}=x_{j}(\bar{t}+0)=x_{k_{i}}(\bar{t}-0), i=1, \ldots, n, \\
& z_{j}^{-}(\bar{t}+0)=z_{k_{1}}^{-}(\bar{t}-0), \\
& z_{k_{i}}^{+}(\bar{t}-0)=z_{k_{i+1}}^{-}(\bar{t}-0), i=1, \ldots, n-1, \\
& z_{k_{n}}^{+}(\bar{t}-0)=z_{j}^{+}(\bar{t}+0) .
\end{aligned}
$$

From $(2.36),(2.37),(2.38)$ and $(2.17)$, we recover conservation of mass

$$
m_{j}(\bar{t}+0)=\sum_{i=1}^{n} m_{k_{i}}(\bar{t}-0)
$$

as expected from (1.3).

We may assume that the $\rho\left(z_{j}^{ \pm}, 0\right), \rho\left(z_{k_{i}}^{ \pm}, 0\right)$ are determined so that $M_{j}(t)=0$ for $t>\bar{t}$ and $M_{k_{i}}(t)=0$ for $t<\bar{t}, i>1, \ldots, n$. Nonetheless there will be an additional contribution in the integral (2.7), arising from the jump discontinuity in $X_{t}(y, t)$ at $t=\bar{t}$ for $z_{j}^{-}(\bar{t})<y<z_{j}^{+}(\bar{t})$.

This additional term in (2.7) may be written

$$
\begin{aligned}
& \lim _{\varepsilon \downarrow 0} \int_{\bar{t}-\varepsilon}^{\bar{t}+\varepsilon} \int_{\mu\left(z_{j}^{-}(\bar{t})\right)}^{\mu\left(z_{j}^{+}(\bar{t})\right)} \theta(X(y, t), t) \zeta\left(X_{t}(y, t)\right)_{t} d \mu(y) d t \\
& =\theta(\bar{x}, \bar{t}) \int_{\mu\left(z_{j}^{-}(\bar{t})\right)}^{\mu\left(z_{j}^{+}(\bar{t})\right)}\left(\zeta\left(X_{t}(y, \bar{t}+0)\right)-\zeta\left(X_{t}(y, \bar{t}-0)\right)\right) d \mu(y) \\
& =\theta(\bar{x}, \bar{t})\left[m_{j}(\bar{t}+0) \zeta\left(s_{j}(\bar{t}+0)\right)-\sum_{i=1}^{n} m_{k_{i}}(\bar{t}-0) \zeta\left(s_{k_{i}}(\bar{t}-0)\right)\right] .
\end{aligned}
$$

It is thus required that the factor in brackets in (2.40) vanish, as expected from (1.4). The vanishing of (2.40) is compatible with (2.39) if and only if the $m_{k}(\bar{t}-0)$ satisfy

$$
\sum_{i=1}^{n} m_{k_{i}}(\bar{t}-0)\left(\zeta\left(s_{j}(\bar{t}+0)\right)-\zeta\left(s_{k_{i}}(\bar{t}-0)\right)\right)=0 .
$$

From the strict convexity of $f$ and (2.1), we have

$$
s_{k_{1}}(\bar{t}-0)>s_{j}(\bar{t}+0)>s_{k_{n}}(\bar{t}-0),
$$

so (2.41) can be achieved by a positive scaling of the $m_{k_{i}}(\bar{t}-0)$, replacing the initial density $\rho(\cdot, 0)$ in the interval $z_{j}^{-}(\bar{t})<y<z_{j}^{+}(\bar{t})$ by

$$
\tilde{\rho}(y, 0)=a_{i} \rho(y, 0), z_{k_{i}}^{-}(\bar{t})<y<z_{k_{i}}^{+}(\bar{t}), i=1, \ldots, n,
$$

with the $a_{i}>0$ chosen so that $(2.41)$ holds with the $m_{k_{i}}(\bar{t}-0)$ replaced by $a_{i} m_{k_{i}}(\bar{t}-0)$. For the case $n=2$, this uniquely determines $a_{2} / a_{1}$; otherwise additional freedom exists. 
Since each of the terms in $M_{k}(t)$ in $(2.28)$ or $(2.29)$ is linear in $m_{k}, \rho\left(z_{k}^{ \pm}, 0\right)$, such a uniform scaling (2.43) on each shock separately will not affect the vanishing of the $M_{k}(t)$.

Thus (2.7) holds for any continuous test function $\theta$ and the proof is complete.

\section{INITIAL DENSITY}

The initial density $\rho(\cdot, 0)$ described in the previous section is by no means unique, but it is fairly easily characterized. In particular, the value of $\rho\left(x_{0}, 0\right)$ is arbitrary for all $x_{0}$ such that the characteristic

$$
x=x_{0}+t f^{\prime}\left(u\left(x_{0}, 0\right)\right), 0<t<\tau,
$$

does not intersect any shock curve.

For $\left(x_{\ell}(\tau), \tau\right)$ a point on a shock curve $\Gamma_{\ell}$, let $L$ denote the connected subset of shock curves (including collision points and points where shocks form spontaneously) containing the point $\left(x_{\ell}(\tau), \tau\right)$. We shall regard $L$ as a tree, working backward in time.

We regard the collision of more than two shocks at a point $Q_{j}$, and the spontaneous formation of a shock with nonzero initial mass $\left(z_{k}^{-}(\underline{t})<z_{k}^{+}(\underline{t})\right.$ for a shock forming at $\left.\left(x_{k}(\underline{t}), \underline{t}\right)\right)$ as exceptional events, as they occur only under special conditions on the initial velocity function $u(\cdot, 0)$.

Theorem 3.1. Assume that the corresponding $m_{k} \in C^{1}\left(I_{k} \rightarrow \mathbb{R}^{+}\right)$for each $\Gamma_{k} \subset$ $L$ is specified. Assume that at points $(\underline{x}, \underline{t}) \in L$ where shocks form spontaneously, they do so with zero initial mass, that $i s, m_{k}(\underline{t}+0)=0$ and $z_{k}^{-}(\underline{t}+0)=z_{k}^{+}(\underline{t}+0)$ where $x_{k}(\underline{t}+0)=\underline{x}$. Assume in addition that only two shocks collide at all collision points $Q_{j} \in L$, and that the limiting masses at the collision points satisfy (2.39) and (2.40).

Then the initial density $\rho(\cdot, 0)$ is uniquely determined in the interval $z_{\ell}^{-}(\tau)<x<$ $z_{\ell}^{+}(\tau)$ by the requirement that the admissible weak solution of (1.5) be compatible with the entropy weak solution of (1.3), (1.4).

Furthermore, the obtained initial density will be strictly positive if and only if

$$
\frac{d m_{k}(t)}{d t}>\frac{m_{k}(t)}{u_{k}^{-}(t)-\zeta\left(s_{k}(t)\right)} \frac{d}{d t} \zeta\left(s_{k}(t)\right)
$$

and

$$
\frac{d m_{k}(t)}{d t}>-\frac{m_{k}(t)}{\zeta\left(s_{k}(t)\right)-u_{k}^{+}(t)} \frac{d}{d t} \zeta\left(s_{k}(t)\right) .
$$

Remark. At point $\left(x_{k}(\underline{t}), \underline{t}\right)$ where a shock forms spontaneously with $z_{k}^{+}(\underline{t})=z_{k}^{-}(\underline{t})$, we require $m_{k}(\underline{t})=0$. Then as $m_{k}(t) /\left(u_{k}^{-}(t)-u_{k}^{+}(t)\right)$ and $d \zeta\left(s_{k}(t)\right) / d t$ are bounded, the conditions (3.2) and (3.3) can always be satisfied. For two shocks colliding at $Q_{j},(2.39)$ and (2.40) uniquely determine the limiting masses of the two incoming shocks depending on the limiting mass of the outgoing shock. Should $n>2$ shocks collide at $Q_{j} \in L$, then as it suffices that shock mass and momentum by conserved at $Q_{j}$ there will be an additional $n-2$ degrees of freedom in $\rho(\cdot, 0)$. And should a shock form spontaneously at $\left(x_{k}(\underline{t}), \underline{t}\right)$ with finite initial mass, i.e. $z_{k}^{-}(\underline{t})<z_{k}^{+}(\underline{t})$ and thus $m_{k}(\underline{t})>0$, then $\rho(\cdot, 0)$ is arbitrary in the interval $z_{k}^{-}(\underline{t})<x<z_{k}^{+}(\underline{t})$, subject only to (2.17). 
Proof. At any point $\left(x_{k}(t), t\right) \in \Gamma_{k}$ (not a collision point) and any $\Gamma_{k} \subset L$, denote

$$
\alpha=\rho_{k}^{-}\left(f^{\prime}\left(u_{k}^{-}\right)-s_{k}\right), \beta=\rho_{k}^{+}\left(s_{k}-f^{\prime}\left(u_{k}^{+}\right)\right) .
$$

Using (1.3), (1.5), the values of $\rho_{k}^{ \pm}$are related to those on the initial line by

$$
\rho_{k}^{ \pm}=\frac{\rho\left(z_{k}^{ \pm}, 0\right)}{1+t f^{\prime \prime}\left(u_{k}^{ \pm}\right) u_{x}\left(z_{k}^{ \pm}, 0\right)}
$$

so that using the entropy condition $(2.2), \rho\left(z_{k}^{ \pm}, 0\right)$ will both be positive if $a, \beta$ are.

Now from $(2.23),(2.26),(2.27)$ and $(3.5)$

$$
\alpha+\beta=\frac{d m_{k}(t)}{d t},
$$

and from $(2.24),(2.26),(2.27)$, and $(3.5)$

$$
\alpha\left(u_{k}^{-}-\zeta\left(s_{k}\right)\right)-\beta\left(\zeta\left(s_{k}\right)-u_{k}^{+}\right)=m_{k}(t) \frac{d \zeta\left(s_{k}(t)\right)}{d t} .
$$

Given $m_{k}$ as a function of $t$ on $\Gamma_{k}$, the right sides of (3.5), (3.6) are determined. The solvability of (3.6), (3.7) for $\alpha, \beta$ is assured by the entropy conditions (2.2), (2.3), and the conditions (3.2), (3.3) are necessary and sufficient for $\alpha, \beta$ so obtained to be positive. This completes the proof.

\section{ACKNOWLEDGMENT}

I wish to thank Professor Erik Aurell for helpful comments on this work.

\section{REFERENCES}

[1] F. Bouchut, "On zero pressure gas dynamics", Advances in kinetic theory and computing, Series on Advances in Mathematics for Applied Sciences v. 122, pp. 171-190, World Scientific, 1994. MR 96e:76107

[2] Y. Brenier and E. Grenier, "Sticky particles and scalar conservation laws", SIAM J. Num. Anal. 35 (1998), pp. 2317-2328. MR 99j:35129

[3] Weinan E, Yu. G. Rykov and Ya. G. Sinai, "Generalized variational principle, global weak solutions and behavior with random initial data systems of conservation laws arising in adhesion particle dynamics", Comm. Math. Physics 177 (1996), pp. 349-380. MR 98a:82077

[4] K. O. Friedrichs and P. D. Lax, "Systems of conservation laws with a convex extension", Proc. Nat. Acad. Sci. USA 68 (1971), pp. 1686-1688. MR 44:3016

[5] B. L. Keyfitz, "Conservation laws, delta shocks and singular shocks", in: "Nonlinear Theory of Generalized Functions", M. Grosser, G. Hormann, M. Kunzinger and M. Oberguggenberger, eds., Chapman and Hall/CRC Press, Boca Raton (1999), pp. 99-111. MR 2000a:00024

[6] D. J. Korchinski, "Solution of a Riemann problem for a 2 X 2 system of conservation laws possessing no classical weak solution", Ph.D. Thesis, Adelphi Univ., Garden City, N. Y. (1977).

[7] P. D. Lax, "Shock waves and entropy", Proc. Sympos. Univ. of Wisconsin (E. H. Zarontouello, ed.) Academic Press, New York (1971), pp. 603-634. MR 52:14677

[8] J. Li and G. Warnecke, "On the uniqueness of entropy solutions to zero pressure gas dynamics," preprint.

[9] J. Li and T. Zhang, "Generalized Rankine-Hugoniot relations of delta-shocks in solution of transportation equations," in: Proc. Int. Conf. PDE, G. Q. Chen, ed., (1997). MR 2000e:35138

[10] M. S. Mock, "Systems of conservation laws of mixed type,", J. Diff. Eq. 37 (1980), pp. 70-88. MR 81m:35088

[11] M. Sever, "An existence theorem in the large for zero pressure gas dynamics", Differential and Integral Equations (to appear). 
[12] M. Sever, "Exchange of conserved quantities, shock loci and Riemann problems", Math. Methods in the Appl. Sci. (to appear).

[13] Y. Zheng, "Systems of conservation laws with incomplete sets of eigenvectors everywhere," in: Advances in Nonlinear Partial Differential Equations and Related Areas, Gui-Qiang Chen et al. eds., World Scientific, Singapore, 1998. MR 2000e:35145

Department of Mathematics, The Hebrew University, Jerusalem, Israel

E-mail address: sever@math.huji.ac.il 